ANNALES

POLONICI MATHEMATICI

$89.2(2006)$

\title{
Generalized method of lines for first order partial functional differential equations
}

\author{
by W. Czernous (Gdańsk)
}

\begin{abstract}
Classical solutions of initial boundary value problems are approximated by solutions of associated differential difference problems. A method of lines for an unknown function for the original problem and for its partial derivatives with respect to spatial variables is constructed. A complete convergence analysis for the method is given. A stability result is proved by using differential inequalities with nonlinear estimates of the Perron type for the given operators.

A discretization in time of the method of lines considered in this paper leads to new difference schemes for the original problem. It is shown by examples that the new method is considerably better than the classical schemes.
\end{abstract}

1. Introduction. For any metric spaces $X$ and $Y$ we denote by $C(X, Y)$ the class of all continuous functions defined on $X$ and taking values in $Y$. We will use vectorial inequalities with the understanding that the same inequalities hold between their corresponding components.

Suppose that $a>0, \tau_{0} \in \mathbb{R}_{+}, \tau \in \mathbb{R}_{+}^{n}, \mathbb{R}_{+}=[0, \infty), b \in \mathbb{R}^{n}, b_{i}>0$ for $1 \leq i \leq n$, are given. Let $c=b+\tau$. We define

$$
\begin{gathered}
E=[0, a] \times(-b, b), \quad E_{0}=\left[-\tau_{0}, 0\right] \times[-c, c], \\
\partial_{0} E=(0, a] \times([-c, c] \backslash(-b, b)), \quad \Omega=E \cup E_{0} \cup \partial_{0} E, \\
D=\left[-\tau_{0}, 0\right] \times[-\tau, \tau] .
\end{gathered}
$$

Suppose that $z: \Omega \rightarrow \mathbb{R}$ and $(t, x) \in \bar{E}$, where $\bar{E}$ is the closure of $E$. We define $z_{(t, x)}: D \rightarrow \mathbb{R}$ as follows:

$$
z_{(t, x)}(\zeta, \xi)=z(t+\zeta, x+\xi), \quad(\zeta, \xi) \in D
$$

Let us denote by $\|\cdot\|_{D}$ the supremum norm in the space $C(D, \mathbb{R})$. Put $\Sigma=\bar{E} \times C(D, \mathbb{R}) \times \mathbb{R}^{n}$ and suppose that

$$
f: \Sigma \rightarrow \mathbb{R}, \quad \varphi: E_{0} \cup \partial_{0} E \rightarrow \mathbb{R}
$$

2000 Mathematics Subject Classification: 35R10, 65M20.

Key words and phrases: differential inequalities, initial boundary value problems, stability and convergence, method of lines. 
and

$$
\alpha_{0}:[0, a] \rightarrow \mathbb{R}, \quad \alpha^{\prime}: \bar{E} \rightarrow \mathbb{R}^{n}, \alpha^{\prime}=\left(\alpha_{1}, \ldots, \alpha_{n}\right),
$$

are given functions. Write $\alpha(t, x)=\left(\alpha_{0}(t), \alpha^{\prime}(t, x)\right)$ for $(t, x) \in \bar{E}$. The requirements on $\alpha$ are that $\alpha(t, x) \in \bar{E}$ for $(t, x) \in E$ and $\alpha_{0}(t) \leq t$ for $t \in[0, a]$. We consider the nonlinear functional differential equation

$$
\partial_{t} z(t, x)=f\left(t, x, z_{\alpha(t, x)}, \partial_{x} z(t, x)\right) \quad \text { on } E
$$

with the initial boundary condition

$$
z(t, x)=\varphi(t, x) \quad \text { on } E_{0} \cup \partial_{0} E,
$$

where $\partial_{x} z=\left(\partial_{x_{1}} z, \ldots, \partial_{x_{n}} z\right)$. We consider classical solutions of (1), (2).

We are interested in establishing a method of approximation of solutions to problem (1), (2) by means of solutions of an associated system of ordinary functional differential equations and in estimating the difference between exact and approximate solutions.

The classical method of lines for partial differential or functional differential equations consists in replacing partial derivatives in spatial variables by difference expressions. Then the original problem is transformed into a system of ordinary differential or functional differential equations.

Results concerning the existence and uniqueness of approximate solutions and the convergence of the numerical method of lines are based on comparison theorems for differential difference inequalities. This method is also used to prove existence theorems for differential or functional differential problems corresponding to parabolic equations or first order hyperbolic systems. The questions mentioned above have an extensive bibliography. It is not our aim to give a full review of papers concerning the method of lines. We only mention the monographs [5], [7], [16] and papers [4], [8], [11], [15], [17], [18]. The book [14] brings a lot of examples of the use of the numerical method of lines.

The papers [1], [12], [13] initiated the investigation of the method of lines for functional differential problems. Parabolic equations with initialboundary conditions and Hamilton-Jacobi equations with initial or initialboundary conditions have been considered. Error estimates implying the convergence of sequences of approximate solutions are proved in these papers by using differential inequalities. The monograph [10] contains an exposition of the theory of the numerical method of lines for hyperbolic functional differential problems.

A new class of numerical methods of lines for (1), (2) is proposed in this paper. Our approach is based on the following idea. We first introduce an additional unknown function $u=\partial_{x} z$ in (1). Then we construct a quasilinear functional differential system with unknown functions $(z, u)$ which is equivalent to the original problem. Finally, we construct a numerical method 
of lines for the unknown functions $(z, u)$ by a discretization in the spatial variables of a new quasilinear differential functional problem.

The paper is organized as follows. In Section 2 we formulate a differential difference problem for (1), (2).

Theorems concerning the method of lines will be based on a comparison result where a function satisfying quasilinear differential difference inequalities is estimated by a solution of a suitable ordinary differential Cauchy problem. The comparison result is proved in Section 3. A convergence theorem and error estimate for the method of lines are presented in Section 4. A difference method which is obtained by a discretization in time of the new numerical method of lines is investigated in Section 5. Numerical examples are given in Section 6 .

Below, we give examples of equations which can be derived from (1) by specifying the operator $f$.

EXAMPLE 1.1. A general class of equations with deviated variables can be obtained in the following way. Suppose that $F: \bar{E} \times \mathbb{R}^{2} \times \mathbb{R}^{n} \rightarrow \mathbb{R}$, $\beta_{0}:[0, a] \rightarrow \mathbb{R}, \beta^{\prime}: E \rightarrow \mathbb{R}^{n}, \beta^{\prime}=\left(\beta_{1}, \ldots, \beta_{n}\right)$, are given functions and

$$
-\tau_{0} \leq \beta_{0}(t)-\alpha_{0}(t) \leq 0, \quad-\tau \leq \beta^{\prime}(t, x)-\alpha^{\prime}(t, x) \leq \tau, \quad(t, x) \in E .
$$

We define the operator $f$ as follows:

$$
\begin{aligned}
& f(t, x, w, q) \\
& \quad F\left(t, x, w\left(-\alpha_{0}(t),-\alpha^{\prime}(t, x)\right), w\left(\beta_{0}(t)-\alpha_{0}(t), \beta^{\prime}(t, x)-\alpha^{\prime}(t, x)\right), q\right)
\end{aligned}
$$

for $(t, x, w, q) \in \Sigma$. Then

$$
f\left(t, x, z_{\alpha(t, x)}, q\right)=F(t, x, z(t, x), z(\beta(t, x)), q),
$$

where $\beta(t, x)=\left(\beta_{0}(t), \beta^{\prime}(t, x)\right)$ and equation (1) is equivalent to the equation with deviated variables

$$
\partial_{t} z(t, x)=F\left(t, x, z(t, x), z(\beta(t, x)), \partial_{x} z(t, x)\right) .
$$

EXAmPle 1.2. Now we consider differential integral equations. Suppose that $\gamma_{0}:[0, a] \rightarrow \mathbb{R}, \gamma^{\prime}: E \rightarrow \mathbb{R}^{n}, \gamma^{\prime}=\left(\gamma_{1}, \ldots, \gamma_{n}\right)$, are given functions and
$-\tau_{0} \leq \gamma_{0}(t)-\alpha_{0}(t) \leq 0$
$-\tau \leq \gamma^{\prime}(t, x)-\alpha^{\prime}(t, x) \leq \tau$,
$(t, x) \in E$.

For given functions $\beta$ satisfying (3) and $F: E \times \mathbb{R}^{2} \times \mathbb{R}^{n} \rightarrow \mathbb{R}$ we define

$$
\begin{aligned}
& f(t, x, w, q) \\
& =F\left(t, x, w\left(-\alpha_{0}(t),-\alpha^{\prime}(t, x)\right), \int_{\beta_{0}(t)-\alpha_{0}(t)}^{\gamma_{0}(t)-\alpha_{0}(t)} \int_{\beta^{\prime}(t, x)-\alpha^{\prime}(t, x)}^{\gamma^{\prime}(t, x)-\alpha^{\prime}(t, x)} w(\tau, y) d y d \tau, q\right)
\end{aligned}
$$


for $(t, x, w, q) \in \Sigma$. Then

$$
f\left(t, x, z_{\alpha(t, x)}, q\right)=F\left(t, x, z(t, x), \int_{\beta(t, x)}^{\gamma(t, x)} z(\tau, y) d y d \tau, q\right)
$$

and (1) reduces to the differential integral equation

$$
\partial_{t} z(t, x)=F\left(t, x, z(t, x), \int_{\beta(t, x)}^{\gamma(t, x)} z(\tau, y) d y d \tau, \partial_{x} z(t, x)\right) .
$$

Existence results for mixed problems can be found in [10, Chapter V].

Let us fix our notations on vectors and matrices. We will denote by $M_{n \times n}$ the space of all $n \times n$ matrices with real elements. For $x, y \in \mathbb{R}^{n}$ and $X \in M_{n \times n}$ where

$$
x=\left(x_{1}, \ldots, x_{n}\right), \quad y=\left(y_{1}, \ldots, y_{n}\right), \quad X=\left[x_{i j}\right]_{i, j=1, \ldots, n}
$$

we put

$$
\begin{gathered}
\|x\|=\max \left\{\left|x_{j}\right|: 1 \leq j \leq n\right\}, \quad x \diamond y=\left(x_{1} y_{1}, \ldots, x_{n} y_{n}\right), \\
\|X\|=\max \left\{\sum_{i=1}^{n}\left|x_{i j}\right|: 1 \leq j \leq n\right\} .
\end{gathered}
$$

The product of two matrices is denoted by " $\star$ ". If $X \in M_{n \times n}$ then $X^{T}$ is the transpose matrix. We use "o" to denote the scalar product in $\mathbb{R}^{n}$.

We denote by $C L(D, \mathbb{R})$ the set of all linear and continuous real functions defined on $C(D, \mathbb{R})$. We denote by $\|\cdot\|_{\star}$ the norm in $C L(D, \mathbb{R})$ generated by the supremum norm in $C(D, \mathbb{R})$. Let $\mathbb{F}(A, B)$ be the class of all functions defined on $A$ and taking values in $B$, for $A$ and $B$ being arbitrary sets. Let $\mathbb{N}$ and $\mathbb{Z}$ be the sets of natural numbers and integers, respectively.

2. Differential difference problems. We define a mesh in $\Omega$ with respect to the spatial variable. Suppose that for $h=\left(h_{1}, \ldots, h_{n}\right)$, where $h_{i}>0$, there exists $N=\left(N_{1}, \ldots, N_{n}\right) \in \mathbb{N}^{n}$ such that $N \diamond h=c$. We denote by $H$ the set of all $h$ having the above property. For $h \in H$ we put $x^{(m)}=m \diamond h$ and $x^{(m)}=\left(x_{1}^{\left(m_{1}\right)}, \ldots, x_{n}^{\left(m_{n}\right)}\right)$ where $m=\left(m_{1}, \ldots, m_{n}\right)$. There is $K \in \mathbb{N}^{n}$ such that $K \diamond h<b \leq(K+1) \diamond h, K+1=\left(K_{1}+1, \ldots, K_{n}+1\right)$. Let

$$
\mathbb{R}_{t . h}^{1+n}=\left\{\left(t, x^{(m)}\right): t \in \mathbb{R}, m \in \mathbb{Z}^{n}\right\} .
$$

We define the sets

$$
\begin{aligned}
E_{0 . h} & =E_{0} \cap \mathbb{R}_{t . h}^{1+n}, & & E_{h}=E \cap \mathbb{R}_{t . h}^{1+n}, \\
\partial_{0} E_{h} & =\partial_{0} E \cap \mathbb{R}_{t . h}^{1+n}, & & \Omega_{h}=\Omega \cap \mathbb{R}_{t . h}^{1+n}
\end{aligned}
$$


and

$$
\Omega_{t . h}=\Omega_{h} \cap\left(\left[-\tau_{0}, t\right] \times \mathbb{R}^{n}\right), \quad-\tau_{0} \leq t \leq a .
$$

Elements of $\Omega$ will be denoted by $\left(t, x^{(m)}\right)$ or $(t, x)$. Let $\mathbb{F}_{\mathrm{c}}\left(\Omega_{h}, \mathbb{R}\right)$ be the set of all functions $w: \Omega_{h} \rightarrow \mathbb{R}$ such that $w(\cdot, x) \in C\left(\left[-\tau_{0}, a\right], \mathbb{R}\right)$ for each fixed $x$. In the same way we define the sets $\mathbb{F}_{\mathrm{c}}\left(E_{h}, \mathbb{R}\right), \mathbb{F}_{\mathrm{c}}\left(E_{0 . h} \cup \partial_{0} E_{h}, \mathbb{R}\right)$. For $z \in \mathbb{F}_{\mathrm{c}}\left(\Omega_{h}, \mathbb{R}\right), u \in \mathbb{F}_{\mathrm{c}}\left(\Omega_{h}, \mathbb{R}^{n}\right)$ and $\left(t, x^{(m)}\right) \in \Omega_{h}$ we write $z^{(m)}(t)=$ $z\left(t, x^{(m)}\right), u^{(m)}(t)=u\left(t, x^{(m)}\right)$ and

$$
\begin{aligned}
& \|z\|_{t . h}=\max \left\{\left|z^{(m)}(\tau)\right|:\left(\tau, x^{(m)}\right) \in \Omega_{t . h}\right\}, \\
& \|u\|_{t . h}=\max \left\{\left\|u^{(m)}(\tau)\right\|:\left(\tau, x^{(m)}\right) \in \Omega_{t . h}\right\} .
\end{aligned}
$$

We need the following assumptions on $f, \varphi$ and $\alpha$.

Assumption $\mathrm{H}_{0}[f, \varphi]$. The function $f: \Sigma \rightarrow \mathbb{R}$ of the variables $(t, x, w, q)$, $x=\left(x_{1}, \ldots, x_{n}\right), q=\left(q_{1}, \ldots, q_{n}\right)$, is continuous and

1) the partial derivatives

$\left(\partial_{x_{1}} f(P), \ldots, \partial_{x_{n}} f(P)\right)=\partial_{x} f(P), \quad\left(\partial_{q_{1}} f(P), \ldots, \partial_{q_{n}} f(P)\right)=\partial_{q} f(P)$ and the Fréchet derivative $\partial_{w} f(P)$ exist for $P=(t, x, w, q) \in \Sigma$ and $\partial_{x} f, \partial_{q} f \in C\left(\Sigma, \mathbb{R}^{n}\right), \partial_{w} f \in C L(D, \mathbb{R})$,

2) there exists $\widetilde{x} \in(-b, b)$ such that

$$
(x-\widetilde{x}) \diamond \partial_{q} f(t, x, w, q) \geq 0 \quad \text { for }(t, x, w, q) \in \Sigma,
$$

3) $\varphi \in C\left(E_{0} \cup \partial_{0} E, \mathbb{R}\right)$ and the partial derivatives $\partial_{x} \varphi$ exist on $E_{0} \cup \partial_{0} E$ and $\partial_{x} \varphi \in C\left(E_{0} \cup \partial_{0} E, \mathbb{R}^{n}\right)$.

Assumption $\mathrm{H}[\alpha]$.

1) The functions $\alpha_{0}:[0, a] \rightarrow[0, a], \alpha^{\prime}: \bar{E} \rightarrow[-b, b]$ are continuous and the partial derivatives $\partial_{x} \alpha^{\prime}(t, x)=\left[\partial_{x_{j}} \alpha_{i}(t, x)\right]_{i, j=1, \ldots, n}$ exist on $\bar{E}$ and $\partial_{x} \alpha^{\prime} \in C\left(\bar{E}, M_{n \times n}\right)$,

2) $\alpha(t, x) \in E$ and $\alpha_{0}(t) \leq t$ for $(t, x) \in E$.

We construct the following numerical method for (1), (2). Let $e_{j} \in \mathbb{R}^{n}$, $1 \leq j \leq n$, be the standard unit vectors. Given $z: \Omega_{h} \rightarrow \mathbb{R}$, let $\delta=$ $\left(\delta_{1}, \ldots, \delta_{n}\right)$ be the difference operator defined by

$$
\begin{array}{ll}
\delta_{j} z^{(m)}(t)=\frac{1}{h_{j}}\left[z^{\left(m+e_{j}\right)}(t)-z^{(m)}(t)\right] & \text { for } x_{j}^{(m)} \geq \widetilde{x}_{j}, \\
\delta_{j} z^{(m)}(t)=\frac{1}{h_{j}}\left[z^{(m)}(t)-z^{\left(m-e_{j}\right)}(t)\right] & \text { for } x_{j}^{(m)}<\widetilde{x}_{j},
\end{array}
$$

for $1 \leq j \leq n$, where $\delta z=\left(\delta_{1} z, \ldots, \delta_{n} z\right)$. Put

We now define the interpolating operator $T_{h}: \mathbb{F}_{\mathrm{c}}\left(\Omega_{h}, \mathbb{R}\right) \rightarrow C(\Omega, \mathbb{R})$.

$$
S=\left\{s=\left(s_{1}, \ldots, s_{n}\right): s_{k} \in\{0,1\} \text { for } 1 \leq k \leq n\right\} .
$$


Suppose that $z: \Omega_{h} \rightarrow \mathbb{R}$ and let $(t, x) \in \Omega$. Then there is $m \in \mathbb{Z}^{n}$ such that $\left(t, x^{(m)}\right),\left(t, x^{(m+1)}\right) \in \Omega_{h}$ and $x^{(m)} \leq x \leq x^{(m+1)}$, where $m+1=$ $\left(m_{1}+1, \ldots, m_{n}+1\right)$. We define

$$
T_{h}[z](t, x)=\sum_{s \in S} z^{(m+s)}(t)\left(\frac{x-x^{(m)}}{h}\right)^{s}\left(1-\frac{x-x^{(m)}}{h}\right)^{1-s}
$$

where

$$
\begin{gathered}
\left(\frac{x-x^{(m)}}{h}\right)^{s}=\prod_{i=1}^{n}\left(\frac{x_{i}-x_{i}^{\left(m_{i}\right)}}{h_{i}}\right)^{s_{i}} \\
\left(1-\frac{x-x^{(m)}}{h}\right)^{1-s}=\prod_{i=1}^{n}\left(1-\frac{x_{i}-x_{i}^{\left(m_{i}\right)}}{h_{i}}\right)^{1-s_{i}}
\end{gathered}
$$

and we take $0^{0}=1$ in the above formulas.

Thus we have defined $T_{h}[z]: \Omega \rightarrow \mathbb{R}$. It is easy to see that $T_{h}[z]$ is continuous on $\Omega$. The above interpolating operator has been first proposed in [10]. If $u \in \mathbb{F}_{\mathrm{c}}\left(\Omega_{h}, \mathbb{R}^{n}\right)$ and $u=\left(u_{1}, \ldots, u_{n}\right)$ then we put $T_{h}[u]=$ $\left(T_{h}\left[u_{1}\right], \ldots, T_{h}\left[u_{n}\right]\right)$.

We will approximate solutions of problem (1), (2) by solutions of a system of ordinary functional differential equations. Let $(z, u) \in \mathbb{F}_{\mathrm{c}}\left(\Omega_{h}, \mathbb{R}^{1+n}\right), u=$ $\left(u_{1}, \ldots, u_{n}\right)$, be unknown functions of the variables $\left(t, x^{(m)}\right) \in \Omega_{h}$. Set

$$
P^{(m)}[z, u](t)=\left(t, x^{(m)},\left(T_{h}[z]\right)_{\alpha\left(t, x^{(m)}\right)}, u^{(m)}(t)\right) .
$$

We will denote by $F_{h}$ and $G_{h}$, the operators defined on $\mathbb{F}_{\mathrm{c}}\left(\Omega_{h}, \mathbb{R}^{1+n}\right)$ in the following way: if $(z, u) \in \mathbb{F}_{\mathrm{c}}\left(\Omega_{h}, \mathbb{R}^{1+n}\right), u=\left(u_{1}, \ldots, u_{n}\right)$, then

$$
\begin{aligned}
& F_{h}[z, u]^{(m)}(t) \\
& \quad=f\left(P^{(m)}[z, u](t)\right)+\partial_{q} f\left(P^{(m)}[z, u](t)\right) \circ\left(\delta z^{(m)}(t)-u^{(m)}(t)\right)
\end{aligned}
$$

and

$$
\begin{aligned}
& G_{h}[z, u]^{(m)}(t) \\
= & \left.\partial_{x} f\left(P^{(m)}[z, u](t)\right)+\partial_{w} f\left(P^{(m)}[z, u](t)\right)\left(T_{h}[u]\right)_{\alpha(t, x}(m)\right) \star \partial_{x} \alpha^{\prime}\left(t, x^{(m)}\right) \\
& +\partial_{q} f\left(P^{(m)}[z, u](t)\right) \star\left[\delta u^{(m)}(t)\right]^{T},
\end{aligned}
$$

where

$$
\begin{aligned}
& \partial_{w} f\left(P^{(m)}[z, u](t)\right)\left(T_{h}[u]\right)_{\alpha\left(t, x^{(m)}\right)} \\
& =\left(\partial_{w} f\left(P^{(m)}[z, u](t)\right)\left(T_{h}\left[u_{1}\right]\right)_{\alpha\left(t, x^{(m)}\right)}, \ldots, \partial_{w} f\left(P^{(m)}[z, u](t)\right)\left(T_{h}\left[u_{n}\right]\right)_{\alpha\left(t, x^{(m)}\right)}\right)
\end{aligned}
$$

and

$$
\delta u^{(m)}(t)=\left[\delta_{j} u_{i}^{(m)}(t)\right]_{i, j=1, \ldots, n} .
$$


We consider the system of ordinary functional differential equations

$$
\begin{aligned}
\frac{d}{d t} z^{(m)}(t) & =F_{h}[z, u]^{(m)}(t), \\
\frac{d}{d t} u^{(m)}(t) & =G_{h}[z, u]^{(m)}(t),
\end{aligned}
$$

for $-K \leq m \leq K$, with the initial boundary condition

$$
z^{(m)}(t)=\varphi_{h}^{(m)}(t), \quad u^{(m)}(t)=\psi_{h}^{(m)}(t) \quad \text { on } E_{0 . h} \cup \partial_{0} E_{h},
$$

and

$$
\varphi_{h}: E_{0 . h} \cup \partial_{0} E_{h} \rightarrow \mathbb{R}, \quad \psi_{h}: E_{0 . h} \cup \partial_{0} E_{h} \rightarrow \mathbb{R}^{n}, \quad \psi_{h}=\left(\psi_{h .1}, \ldots, \psi_{h . n}\right),
$$

are given functions. We prove that under natural assumptions on given functions, classical solutions of (1), (2) can be approximated by solutions of (13)-(15).

The differential difference system (13)-(14) with initial boundary condition (15) is called a generalized method of lines for problem (1), (2). This method is obtained in the following way.

We transform the nonlinear problem (1), (2) into a system of quasilinear differential equations with unknown functions $(z, u)$ where $u=\partial_{x} z$. Suppose that Assumptions $\mathrm{H}_{0}[f, \varphi]$ and $\mathrm{H}[\alpha]$ are satisfied. Write

$$
U[z, u ; t, x]=\left(t, x, z_{\alpha(t, x)}, u(t, x)\right) .
$$

For $u: \Omega \rightarrow \mathbb{R}^{n}$ and for $(t, x) \in \bar{E}$ set

$$
\partial_{w} f(P) u_{\alpha(t, x)}=\left(\partial_{w} f(P)\left(u_{1}\right)_{\alpha(t, x)}, \ldots, \partial_{w} f(P)\left(u_{n}\right)_{\alpha(t, x)}\right)
$$

where $P=(t, x, w, q) \in \Sigma$. We consider the quasilinear functional differential system

$$
\begin{aligned}
\partial_{t} z(t, x)= & f(U[z, u ; t, x])+\partial_{q} f(U[z, u ; t, x]) \circ\left(\partial_{x} z(t, x)-u(t, x)\right), \\
\partial_{t} u(t, x)= & \partial_{x} f(U[z, u ; t, x])+\partial_{w} f(U[z, u ; t, x]) u_{\alpha(t, x)} \star \partial_{x} \alpha^{\prime}(t, x) \\
& +\partial_{q} f(U[z, u ; t, x]) \star\left[\partial_{x} u(t, x)\right]^{T}
\end{aligned}
$$

with the initial boundary condition

$$
z(t, x)=\varphi(t, x), \quad u(t, x)=\partial_{x} \varphi(t, x) \quad \text { on } E_{0} \cup \partial_{0} E .
$$

Note that each equation of system (16), (17) depends on the unknown functions $(z, u)$ and it contains partial derivatives of only one scalar function. System (16), (17) also has the following property: the differential equations of bicharacteristics for (16) and for (17) are the same and they have the form

$$
\eta^{\prime}(t)=-\partial_{q} f\left(t, \eta(t), z_{\alpha(t, \eta(t))}, u(t, \eta(t))\right) .
$$

This property of system (16), (17) is important in the investigation of the stability of the differential difference problem (13)-(15). 
Under natural assumptions on the given functions the above problem has the following property: if $(\widetilde{z}, \widetilde{u})$ is a solution of $(16)-(18)$ then $\partial_{x} \widetilde{z}=\widetilde{u}$, and the conditions

(A) $v: \Omega \rightarrow \mathbb{R}$ is a classical solution of (1), (2),

(B) $\left(v, \partial_{x} v\right)$ are a classical solution of (16)-(18),

are equivalent.

The differential difference problem (13)-(15) is a discretization with respect to the spatial variables of (16)-(18). The above method of quasilinearization was introduced and studied in a non-functional setting by S. Cinquini and M. Cinquini-Cibrario in [2], [3]. They used this method in the existence and uniqueness theory for generalized solutions of (1), (2). This method is also adopted for nonlinear functional differential problems in [10].

3. Differential difference inequalities. For a function $g \in \mathbb{F}_{\mathrm{c}}\left(\Omega_{h}, \mathbb{R}^{k}\right)$, $g=\left(g_{1}, \ldots, g_{k}\right)$, we write

$$
g^{(m)}(t)=g\left(t, x^{(m)}\right), \quad \delta g^{(m)}(t)=\left[\delta_{j} g_{i}^{(m)}(t)\right]_{i=1, \ldots, k, j=1, \ldots, n}
$$

and

$$
D_{-} g^{(m)}(t)=\left(D_{-} g_{1}^{(m)}(t), \ldots, D_{-} g_{k}^{(m)}(t)\right)
$$

where $D_{-} g_{i}^{(m)}(t)$ is the left hand Dini derivative of $g_{i}^{(m)}(\cdot)$ at the point $t$. The norm in $\mathbb{R}^{k}$ is defined by

$$
\|p\|_{0}=\max \left\{\left|p_{i}\right|: 1 \leq i \leq k\right\}
$$

where $p=\left(p_{1}, \ldots, p_{k}\right)$. For $g \in \mathbb{F}_{\mathrm{c}}\left(\Omega_{h}, \mathbb{R}^{k}\right)$ we put

$$
\|g\|_{t . h}=\max \left\{\left\|g^{(m)}(\tau)\right\|_{0}:\left(\tau, x^{(m)}\right) \in \Omega_{t . h}\right\}, \quad 0 \leq t \leq a .
$$

Let us now state a comparison theorem which will be used in the following.

TheOREM 3.1. Suppose that

1) $\Lambda: \bar{E} \times \mathbb{F}_{\mathrm{c}}\left(\Omega_{h}, \mathbb{R}^{k}\right) \rightarrow \mathbb{R}^{n}, \Lambda=\left(\lambda_{1}, \ldots, \lambda_{n}\right)$, satisfies the conditions

$$
(x-\widetilde{x}) \diamond \Lambda(t, x, w) \geq 0 \quad \text { for }(t, x, w) \in \bar{E} \times \mathbb{F}_{\mathrm{c}}\left(\Omega_{h}, \mathbb{R}^{k}\right),
$$

2) $\sigma:[0, a] \times \mathbb{R}_{+} \rightarrow \mathbb{R}_{+}$is continuous and it is nondecreasing with respect to the second variable,

3) $\psi \in \mathbb{F}_{\mathrm{c}}\left(\Omega_{h}, \mathbb{R}^{k}\right), \psi=\left(\psi_{1}, \ldots, \psi_{k}\right)$, satisfies the differential difference inequality

$$
\left\|D_{-} \psi^{(m)}(t)-\Lambda\left(t, x^{(m)}, \psi\right) \star\left[\delta \psi^{(m)}(t)\right]^{T}\right\|_{0} \leq \sigma\left(t,\|\psi\|_{t . h}\right)
$$

on $E_{h}$ and the initial-boundary inequality

$$
\left\|\psi^{(m)}(t)\right\|_{0} \leq \eta, \quad\left(t, x^{(m)}\right) \in E_{0 . h} \cup \partial_{0} E_{h},
$$


4) there exists on $[0, a]$ the maximum solution $\omega(\cdot, \eta)$ of the Cauchy problem

$$
\omega^{\prime}(t)=\sigma(t, \omega(t)), \quad \omega(0)=\eta .
$$

Under these assumptions we have

$$
\left\|\psi^{(m)}(t)\right\|_{0} \leq \omega(t, \eta) \quad \text { on } E_{h} .
$$

Proof. For $\varepsilon>0$ we denote by $\omega(t, \eta, \varepsilon)$ the maximum solution of the Cauchy problem

$$
\omega^{\prime}(t)=\sigma(t, \omega(t))+\varepsilon, \quad \omega(0)=\eta+\varepsilon .
$$

We can see that there exists $\varepsilon_{0}>0$ such that for $0<\varepsilon<\varepsilon_{0}$ the solution $\omega(\cdot, \eta, \varepsilon)$ is defined on $[0, a]$ and $\lim _{\varepsilon \rightarrow 0} \omega(t, \eta, \varepsilon)=\omega(t, \eta)$ uniformly on $[0, a]$. We prove that

$$
\left\|\psi^{(m)}(t)\right\|_{0}<\omega(t, \eta, \varepsilon) \quad \text { for }\left(t, x^{(m)}\right) \in \Omega_{h} .
$$

Suppose by contradiction that (22) fails to be true. Then the set

$$
J_{+}=\left\{t \in[0, a):\left\|\psi^{(m)}(t)\right\|_{0} \geq \omega(t, \eta, \varepsilon) \text { for some } x^{(m)} \in[-c, c]\right\}
$$

is not empty. If we put $t^{*}=\min J_{+}$, from (20) it is clear that $t^{*}>0$ and there exists $x^{(\widetilde{m})} \in[-c, c]$ such that

$$
\left\|\psi^{(\widetilde{m})}\left(t^{*}\right)\right\|_{0}=\omega\left(t^{*}, \eta, \varepsilon\right) .
$$

From (20) it follows that $\left(t^{*}, x^{(\widetilde{m})}\right) \in E_{h}$. We see that there is $i, 1 \leq i \leq k$, such that $\left\|\psi^{(\widetilde{m})}\left(t^{*}\right)\right\|_{0}=\left|\psi_{i}^{(\widetilde{m})}\left(t^{*}\right)\right|$. Note that $\|\psi\|_{t^{*} . h}=\omega\left(t^{*}, \eta, \varepsilon\right)$. Two possibilities can happen: either (i) $\psi_{i}^{(\widetilde{m})}\left(t^{*}\right)=\omega\left(t^{*}, \eta, \varepsilon\right)$ or (ii) $\psi_{i}^{(\widetilde{m})}\left(t^{*}\right)=$ $-\omega\left(t^{*}, \eta, \varepsilon\right)$. If (i) holds, then for $t \in\left[0, t^{*}\right)$ we have

$$
\frac{\psi_{i}^{(\widetilde{m})}(t)-\psi_{i}^{(\widetilde{m})}\left(t^{*}\right)}{t-t^{*}}>\frac{\omega(t, \eta, \varepsilon)-\omega\left(t^{*}, \eta, \varepsilon\right)}{t-t^{*}} .
$$

Thus

$$
D_{-} \psi_{i}^{(\widetilde{m})}\left(t^{*}\right) \geq \omega^{\prime}\left(t^{*}, \eta, \varepsilon\right)
$$

\section{Write}

$$
A_{+}[\widetilde{m}]=\left\{j: 1 \leq j \leq n \text { and } x_{j}^{\left(\widetilde{m}_{j}\right)} \geq \widetilde{x}_{j}\right\}, \quad A_{-}[\widetilde{m}]=\{1, \ldots, n\} \backslash A_{+}[\widetilde{m}] .
$$

Then

$$
\begin{aligned}
\sum_{j=1}^{n} \lambda_{j}\left(t^{*}, x^{(\widetilde{m})}, \psi\right) \delta_{j} \psi_{i}^{(\widetilde{m})}\left(t^{*}\right) & \\
= & \sum_{j \in A_{+}[\widetilde{m}]} \lambda_{j}\left(t^{*}, x^{(\widetilde{m})}, \psi\right) \frac{1}{h_{j}}\left[\psi_{i}^{\left(\widetilde{m}+e_{j}\right)}\left(t^{*}\right)-\psi_{i}^{(\widetilde{m})}\left(t^{*}\right)\right] \\
& +\sum_{j \in A_{-}[\tilde{m}]} \lambda_{j}\left(t^{*}, x^{(\widetilde{m})}, \psi\right) \frac{1}{h_{j}}\left[\psi_{i}^{(\widetilde{m})}\left(t^{*}\right)-\psi_{i}^{\left(\widetilde{m}-e_{j}\right)}\left(t^{*}\right)\right] \leq 0 .
\end{aligned}
$$


According to (19) and (24), we have

$$
\begin{aligned}
D_{-} \psi_{i}^{(\widetilde{m})}\left(t^{*}\right) & \leq \sum_{j=1}^{n} \lambda_{j}\left(t^{*}, x^{(\widetilde{m})}, \psi\right) \delta_{j} \psi_{i}^{(\widetilde{m})}\left(t^{*}\right)+\sigma\left(t^{*},\|\psi\|_{t^{*} . h}\right) \\
& \leq \sigma\left(t^{*}, \omega\left(t^{*}, \eta, \varepsilon\right)\right)<\sigma\left(t^{*}, \omega\left(t^{*}, \eta, \varepsilon\right)\right)+\varepsilon=\omega^{\prime}\left(t^{*}, \eta, \varepsilon\right),
\end{aligned}
$$

which contradicts (23). Case (ii) is treated analogously. Thus (22) is proved. It follows from (22) that

$$
\left\|\psi^{(m)}(t)\right\|_{0}<\omega(t, \eta, \varepsilon) \quad \text { for }\left(t, x^{(m)}\right) \in E_{h}
$$

where $0<\varepsilon<\varepsilon_{0}$ is arbitrary. Letting $\varepsilon$ tend to 0 yields (21).

REMARK 3.2. Differential difference inequalities generated by mixed problems for first order partial functional differential equations were considered in [10, Chapter 6]. Here are the differences between our results and Theorem 6.6 of [10].

1. There are differential equations with deviated variables and differential integral problems for which our comparison result can be used and Theorem 6.6 from [10] is not applicable. This is due to the fact that the Hale operator $(t, x) \mapsto z_{(t, x)}$ considered in our paper is more general than the corresponding operator in [10]:

2. It is assumed in [10] that $\lambda_{i}(t, x, w) \geq 0$ for $1 \leq i \leq \kappa$ and $\lambda_{i}(t, x, w) \leq$ 0 for $\kappa+1 \leq i \leq n$ where $0 \leq \kappa \leq n$ is fixed. This requirement is replaced by condition 1) in Theorem 3.1 .

4. Convergence of the method of lines. We will need the following property of the interpolating operator $T_{h}$.

Lemma 4.1. Suppose that

1) $\widetilde{v}: \Omega \rightarrow \mathbb{R}$ is of class $C^{1}$ and $\widetilde{v}_{h}$ is the restriction of $\widetilde{v}$ to $\Omega_{h}$,

2) $h \in H$ and $\widetilde{c} \in \mathbb{R}_{+}$is a constant such that

Then

$$
\left|\partial_{x_{i}} \widetilde{v}(t, x)\right| \leq \widetilde{c}, \quad 1 \leq i \leq n,(t, x) \in \Omega .
$$

$$
\max \left\{\left|T_{h}\left[\widetilde{v}_{h}\right](t, x)-\widetilde{v}(t, x)\right|:(t, x) \in \Omega\right\} \leq \widetilde{c}\|h\| .
$$

The proof of the above lemma is similar to the proof of Theorem 5.27 in [10]. We omit the details.

We next formulate further assumptions on the given functions.

Assumption $\mathrm{H}[\sigma]$. The function $\sigma:[0, a] \times \mathbb{R}_{+} \rightarrow \mathbb{R}_{+}$is continuous and

1) $\sigma$ is nondecreasing with respect to the second variable,

2) for each $c \in \mathbb{R}_{+}$and $d \geq 1$ the maximal solution of the Cauchy problem

$$
\omega^{\prime}(t)=c \omega(t)+d \sigma(t, \omega(t)), \quad \omega(0)=0,
$$

is $\omega(t)=0, t \in[0, a]$. 
Assumption $\mathrm{H}[f, \varphi]$. Assumption $\mathrm{H}_{0}[f, \varphi]$ is satisfied and

1) there is $B \in \mathbb{R}_{+}$such that

$$
\left\|\partial_{x} f(P)\right\|,\left\|\partial_{w} f(P)\right\|_{\star},\left\|\partial_{q} f(P)\right\| \leq B \quad \text { for all } P=(t, x, w, q) \in \Sigma,
$$

2) there is a function $\sigma:[0, a] \times \mathbb{R}_{+} \rightarrow \mathbb{R}_{+}$such that Assumption $\mathrm{H}[\sigma]$ is satisfied and the terms

$$
\begin{gathered}
\left\|\partial_{x} f(t, x, w, q)-\partial_{x} f(t, x, \bar{w}, \bar{q})\right\|, \\
\left\|\partial_{w} f(t, x, w, q)-\partial_{w} f(t, x, \bar{w}, \bar{q})\right\|_{\star}, \quad\left\|\partial_{q} f(t, x, w, q)-\partial_{q} f(t, x, \bar{w}, \bar{q})\right\|
\end{gathered}
$$

are bounded from above by $\sigma\left(t, \max \left\{\|w-\bar{w}\|_{D},\|q-\bar{q}\|\right\}\right)$,

3) $\varphi: E_{0} \cup \partial_{0} E \rightarrow \mathbb{R}$ is of class $C^{2}$.

Lemma 4.2. If Assumptions $\mathrm{H}[\alpha]$ and $\mathrm{H}[f, \varphi]$ are satisfied then there exists a unique solution $\left(z_{h}, u_{h}\right): \Omega_{h} \rightarrow \mathbb{R}^{1+n}, u_{h}=\left(u_{h .1}, \ldots, u_{h . n}\right)$, of problem (13)-(15).

Proof. The right hand sides of (13)-(15) are continuous. Hence a solution $\left(z_{h}, u_{h}\right)$ of $(13)-(15)$ exists on $\Omega_{h} \cap\left([0, \widetilde{a}) \times \mathbb{R}^{n}\right)$ for some $\widetilde{a} \in[0, a]$. It follows from Assumptions $\mathrm{H}[\alpha], \mathrm{H}[f, \varphi]$ that there exist $A, C \in \mathbb{R}_{+}$such that

$\left|\frac{d}{d t} z_{h}^{(m)}(t)-\partial_{q} f\left(P^{(m)}\left[z_{h}, u_{h}\right](t)\right) \circ \delta z_{h}^{(m)}(t)\right| \leq A \max \left\{\left\|z_{h}\right\|_{t . h},\left\|u_{h}\right\|_{t . h}\right\}+C$

and

$\left\|\frac{d}{d t} u_{h}^{(m)}(t)-\partial_{q} f\left(P^{(m)}\left[z_{h}, u_{h}\right](t)\right) \star\left[\delta u_{h}^{(m)}(t)\right]^{T}\right\| \leq A \max \left\{\left\|z_{h}\right\|_{t . h},\left\|u_{h}\right\|_{t . h}\right\}+C$.

Applying Theorem 3.1 with

$$
\begin{gathered}
\sigma(t, p)=A p+C, \quad \eta=\max \left\{\eta_{0}, \eta_{1}\right\}, \\
\eta_{0}=\max \left\{\left|\varphi_{h}^{(m)}(t)\right|:\left(t, x^{(m)}\right) \in E_{0 . h} \cup \partial_{0} E_{h}\right\}, \\
\eta_{1}=\max \left\{\left\|\psi_{h}^{(m)}(t)\right\|:\left(t, x^{(m)}\right) \in E_{0 . h} \cup \partial_{0} E_{h}\right\}
\end{gathered}
$$

we get

$$
\max \left\{\left\|z_{h}\right\|_{t . h},\left\|u_{h}\right\|_{t . h}\right\} \leq \eta e^{A t}+\frac{C}{A}\left(e^{A t}-1\right), \quad t \in[0, \widetilde{a}) .
$$

Now the classical theorems on the existence of solutions of Cauchy problems for systems of ordinary differential equations show that the solution $\left(z_{h}, u_{h}\right)$ is defined on $\Omega_{h}$.

Now we prove that the solution is unique. Let $\left(z_{h}, u_{h}\right): \Omega_{h} \rightarrow \mathbb{R}^{1+n}$ and $\left(\widetilde{z}_{h}, \widetilde{u}_{h}\right): \Omega_{h} \rightarrow \mathbb{R}^{1+n}$ be two solutions of (13)-(15). Write

$$
\bar{z}_{h}=z_{h}-\widetilde{z}_{h}, \quad \bar{u}_{h}=u_{h}-\widetilde{u}_{h} .
$$


It follows from Assumptions $\mathrm{H}[\alpha], \mathrm{H}[f, \varphi]$ that there are $\bar{A} \in \mathbb{R}_{+}$and $\bar{B} \geq 1$ such that

$$
\begin{aligned}
\mid \frac{d}{d t} \bar{z}_{h}^{(m)}(t)- & \partial_{q} f\left(P^{(m)}\left[z_{h}, u_{h}\right](t)\right) \circ \delta \bar{z}_{h}^{(m)}(t) \mid \\
& \leq \bar{A} \max \left\{\left\|\bar{z}_{h}\right\|_{t . h},\left\|\bar{u}_{h}\right\|_{t . h}\right\}+\bar{B} \sigma\left(t, \max \left\{\left\|\bar{z}_{h}\right\|_{t . h},\left\|\bar{u}_{h}\right\|_{t . h}\right\}\right)
\end{aligned}
$$

and

$$
\begin{aligned}
\| \frac{d}{d t} \bar{u}_{h}^{(m)}(t) & -\partial_{q} f\left(P^{(m)}\left[z_{h}, u_{h}\right](t)\right) \star\left[\delta \bar{u}_{h}^{(m)}(t)\right]^{T} \| \\
& \leq \bar{A} \max \left\{\left\|\bar{z}_{h}\right\|_{t . h},\left\|\bar{u}_{h}\right\|_{t . h}\right\}+\bar{B} \sigma\left(t, \max \left\{\left\|\bar{z}_{h}\right\|_{t . h},\left\|\bar{u}_{h}\right\|_{t . h}\right\}\right)
\end{aligned}
$$

for $\left(t, x^{(m)}\right) \in E_{h}$. Moreover, $\bar{z}_{h}^{(m)}(t)=0$ and $\bar{u}_{h}^{(m)}(t)=0$ for $\left(t, x^{(m)}\right) \in$ $E_{0 . h} \cup \partial_{0} E_{h}$. Then, by Theorem 3.1 we obtain $\bar{z}_{h}=0$ and $\bar{u}_{h}=0$ on $\Omega_{h}$, which completes the proof.

Now we prove a theorem on convergence of a generalized method of lines.

TheOrem 4.3. Suppose that Assumptions $\mathrm{H}[\alpha], \mathrm{H}[f, \varphi]$ are satisfied and

1) $\left(z_{h}, u_{h}\right)$, where $z_{h}: \Omega_{h} \rightarrow \mathbb{R}, u_{h}: \Omega_{h} \rightarrow \mathbb{R}^{n}, u_{h}=\left(u_{h .1}, \ldots, u_{h . n}\right)$, are the unique solution of problem (13)-(15) with $\delta$ given by (9), (10),

2) $v: \Omega \rightarrow \mathbb{R}$ is the solution of problem (1), (2) and $v$ is of class $C^{2}$ on $\Omega$,

3) $\left(v_{h}, w_{h}\right)$, where $w_{h}=\left(w_{1 . h}, \ldots, w_{n . h}\right)$, are the restrictions of $\left(v, \partial_{x} v\right)$, respectively, to $\Omega_{h}$,

4) there is a function $\beta_{0}: H \rightarrow \mathbb{R}_{+}$such that

$$
\begin{aligned}
& \max \left\{\left|\left(\varphi_{h}-\varphi\right)^{(m)}(t)\right|,\left\|\left(\psi_{h}-\partial_{x} \varphi\right)^{(m)}(t)\right\|\right\} \leq \beta_{0}(h) \text { on } E_{0 . h} \cup \partial_{0} E_{h} \\
& \text { and } \lim _{h \rightarrow 0} \beta_{0}(h)=0 .
\end{aligned}
$$

Then there is a number $\varepsilon_{0}>0$ and a function $\beta: H \rightarrow \mathbb{R}_{+}$such that for $\|h\| \leq \varepsilon_{0}$,

$$
\max \left\{\left|\left(z_{h}-v_{h}\right)^{(m)}(t)\right|,\left\|\left(u_{h}-w_{h}\right)^{(m)}(t)\right\|\right\} \leq \beta(h) \quad \text { on } E_{h}
$$

and $\lim _{h \rightarrow 0} \beta(h)=0$.

Proof. We will write differential difference inequalities for the functions $z_{h}-v_{h}$ and $u_{h}-w_{h}$. We insert the functions $\left(v_{h}, w_{h}\right)$ into system (13)-(15). We define $\Gamma_{h}: \Omega_{h} \rightarrow \mathbb{R}, U_{h}: \Omega_{h} \rightarrow \mathbb{R}^{n}$ in the following way:

$$
\begin{aligned}
\frac{d}{d t} v_{h}^{(m)}(t) & =F_{h}\left[v_{h}, w_{h}\right]^{(m)}(t)-\Gamma_{h}^{(m)}(t), \\
\frac{d}{d t} w_{h}^{(m)}(t) & =G_{h}\left[v_{h}, w_{h}\right]^{(m)}(t)-U_{h}^{(m)}(t) .
\end{aligned}
$$

Let $\widetilde{c}, \widetilde{s} \in \mathbb{R}_{+}$be constants such that 


$$
\left|\partial_{t} v(t, x)\right|,\left|\partial_{x_{i}} v(t, x)\right|,\left|\partial_{t t} v(t, x)\right| \leq \widetilde{c}
$$

$$
\left|\partial_{x_{i} x_{j}} v(t, x)\right|,\left|\partial_{t x_{i}} v(t, x)\right| \leq \widetilde{c}
$$

for $(t, x) \in \Omega, i, j=1, \ldots, n$, and

$$
\left\|\partial_{x} \alpha^{\prime}(t, x)\right\| \leq \widetilde{s}, \quad(t, x) \in \bar{E}
$$

It follows from Lemma 4.1, Assumption $\mathrm{H}[f, \varphi]$ and condition 2) of the theorem that there is $\gamma: H \rightarrow \mathbb{R}_{+}$such that

$$
\max \left\{\left|\Gamma_{h}^{(m)}(t)\right|,\left\|U_{h}^{(m)}(t)\right\|\right\} \leq \gamma(h) \quad \text { on } E_{h} \quad \text { and } \quad \lim _{h \rightarrow 0} \gamma(h)=0 .
$$

It follows from (13), (14), (16), (17), (26), (27) that the functions $z_{h}-v_{h}$ and $u_{h}-w_{h}$ satisfy the differential difference equations

$$
\begin{aligned}
\frac{d}{d t}\left(z_{h}-v_{h}\right)^{(m)}(t)= & f\left(P^{(m)}\left[z_{h}, u_{h}\right](t)\right)-f\left(P^{(m)}\left[v_{h}, w_{h}\right](t)\right) \\
& +\partial_{q} f\left(P^{(m)}\left[z_{h}, u_{h}\right](t)\right) \circ\left(\delta z_{h}^{(m)}(t)-u_{h}^{(m)}(t)\right) \\
& -\partial_{q} f\left(P^{(m)}\left[v_{h}, w_{h}\right](t)\right) \circ\left(\delta v_{h}^{(m)}(t)-w_{h}^{(m)}(t)\right)+\Gamma_{h}^{(m)}(t),
\end{aligned}
$$

and

$$
\begin{aligned}
\frac{d}{d t}\left(u_{h}-w_{h}\right)^{(m)}(t)= & \partial_{x} f\left(P^{(m)}\left[z_{h}, u_{h}\right](t)\right)-\partial_{x} f\left(P^{(m)}\left[v_{h}, w_{h}\right](t)\right) \\
& +\partial_{w} f\left(P^{(m)}\left[z_{h}, u_{h}\right](t)\right)\left(T_{h}\left[u_{h}\right]\right)_{\alpha(t, x(m))} \star \partial_{x} \alpha^{\prime(m)}(t) \\
& \left.-\partial_{w} f\left(P^{(m)}\left[v_{h}, w_{h}\right](t)\right)\left(T_{h}\left[w_{h}\right]\right)_{\alpha(t, x}(m)\right) \star \partial_{x} \alpha^{\prime(m)}(t) \\
& +\partial_{q} f\left(P^{(m)}\left[z_{h}, u_{h}\right](t)\right) \star\left[\delta u_{h}^{(m)}(t)\right]^{T} \\
& -\partial_{q} f\left(P^{(m)}\left[v_{h}, w_{h}\right](t)\right) \star\left[\delta w_{h}^{(m)}(t)\right]^{T}+U_{h}^{(m)}(t)
\end{aligned}
$$

for $\left(t, x^{(m)}\right) \in E_{h}$. Write

$$
\zeta_{h}(t)=\max \left\{\left\|z_{h}-v_{h}\right\|_{t . h},\left\|u_{h}-w_{h}\right\|_{t . h}\right\}, \quad t \in[0, a] .
$$

According to Assumption $\mathrm{H}[f, \varphi]$ we have

$$
\left|f\left(P^{(m)}\left[z_{h}, u_{h}\right](t)\right)-f\left(P^{(m)}\left[v_{h}, w_{h}\right](t)\right)\right| \leq B(n+1) \zeta_{h}(t) .
$$

In the same manner we can see that

$$
\left\|\partial_{q} f\left(P^{(m)}\left[z_{h}, u_{h}\right](t)\right)-\partial_{q} f\left(P^{(m)}\left[v_{h}, w_{h}\right](t)\right)\right\| \leq \sigma\left(t, \zeta_{h}(t)\right)
$$

for $\left(t, x^{(m)}\right) \in E_{h}$. It is easily seen that the conclusion analogous to (31) can be drawn for the derivatives $\partial_{x} f, \partial_{w} f$. According to the above estimates, we have the following differential difference inequalities:

$$
\begin{aligned}
\mid \frac{d}{d t}\left(z_{h}-v_{h}\right)^{(m)}(t)-\partial_{q} f\left(P^{(m)}\left[z_{h}, u_{h}\right](t)\right) & \circ \delta\left(z_{h}-v_{h}\right)^{(m)}(t) \mid \\
& \leq \bar{c} \zeta_{h}(t)+\bar{d} \sigma\left(t, \zeta_{h}(t)\right)+\gamma(h)
\end{aligned}
$$


and

$$
\begin{array}{r}
\left\|\frac{d}{d t}\left(u_{h}-w_{h}\right)^{(m)}(t)-\partial_{q} f\left(P^{(m)}\left[z_{h}, u_{h}\right](t)\right) \star\left[\delta\left(u_{h}-w_{h}\right)^{(m)}(t)\right]^{T}\right\| \\
\leq \bar{c} \zeta_{h}(t)+\bar{d} \sigma\left(t, \zeta_{h}(t)\right)+\gamma(h),
\end{array}
$$

where

$$
\bar{c}=B \bar{s}, \quad \bar{d}=\max \{2 n \widetilde{c}, 1+\widetilde{c s}+n \widetilde{c}\}, \quad \bar{s}=\max \{2 n+1, \widetilde{s}\} .
$$

Consider the Cauchy problem

$$
\omega^{\prime}(t)=\bar{c} \omega(t)+\bar{d} \sigma(t, \omega(t))+\gamma(h), \quad \omega(0)=\beta_{0}(h) .
$$

It follows from Assumption $\mathrm{H}[f, \varphi]$ that there is $\varepsilon_{0}>0$ such that for $\|h\| \leq \varepsilon_{0}$ there exists the maximum solution $\omega_{h}$ of $(33)$ and $\omega_{h}$ is defined on $[0, a]$. Applying Theorem 3.1 with the function $\widetilde{\sigma}(t, \omega)=\bar{c} \omega+\bar{d} \sigma(t, \omega)+\gamma(h)$ on the right hand side of (19), we get the estimate

$$
\zeta_{h}(t) \leq \omega_{h}(t) \quad \text { for }\left(t, x^{(m)}\right) \in E_{h} .
$$

Moreover, $\lim _{h \rightarrow 0} \omega_{h}(t)=0$ uniformly on $[0, a]$. Thus we get $(25)$ for $\beta(h)=$ $\omega_{h}(a)$. This completes the proof.

We now give an error estimate for a generalized method of lines.

Lemma 4.4. Suppose that all the assumptions of Theorem 4.3 are satisfied with $\sigma(t, \tau)=L \tau$ on $[0, a] \times \mathbb{R}_{+}$for some $L \in \mathbb{R}_{+}$. Then we have the following error estimate of method (13)-(15):

$$
\max \left\{\left\|\left(z_{h}-v_{h}\right)\right\|_{t . h},\left\|\left(u_{h}-w_{h}\right)\right\|_{t . h}\right\} \leq \bar{\beta}(h) \quad \text { on } E_{h}
$$

where

$$
\bar{\beta}(h)= \begin{cases}\beta_{0}(h) e^{\widetilde{L} a}+\gamma(h) \frac{e^{\widetilde{L} a}-1}{\widetilde{L}} & \text { if } \widetilde{L}>0, \\ \beta_{0}(h)+a \gamma(h) & \text { if } \widetilde{L}=0,\end{cases}
$$

where $\widetilde{L}=\bar{c}+L \bar{d}$ and $\bar{c}, \bar{d}$ are given by (28), (29), (32).

Proof. Since the estimate (30) is satisfied, we obtain the assertion from (34) and (25) and by solving problem (33).

Note that in Lemma 4.4 we have assumed that the functions $\partial_{w} f, \partial_{q} f$ satisfy the Lipschitz condition with respect to $(w, q)$ on $\Sigma$.

\section{Difference methods generated by a generalized method of} lines. We define a mesh on $\left[-\tau_{0}, a\right]$ in the following way. Let $h_{0}$ be the step of the mesh and $t^{(r)}=r h_{0}, r \in \mathbb{Z}$, be the nodal points. Denote by $H^{\prime}$ the set of all $h^{\prime}=\left(h_{0}, h\right)$ such that $h \in H$ and there is $N_{0} \in \mathbb{Z}$ with $N_{0} h_{0}=\tau_{0}$. There is $K_{0} \in \mathbb{N}$ such that $K_{0} h_{0} \leq a<\left(K_{0}+1\right) h_{0}$. For $h^{\prime} \in H^{\prime}$ we put 


$$
\begin{aligned}
& \left\|h^{\prime}\right\|=h_{0}+h_{1}+\cdots+h_{n} \text { and } \\
& \quad \mathbb{R}_{h^{\prime}}^{1+n}=\left\{\left(t^{(r)}, x^{(m)}\right):(r, m) \in \mathbb{Z}^{1+n}\right\}, \quad I_{h^{\prime}}=\left\{t^{(r)}: 0 \leq r \leq K_{0}\right\}
\end{aligned}
$$

and

$$
\begin{aligned}
E_{0 . h^{\prime}} & =E_{0} \cap \mathbb{R}_{h^{\prime}}^{1+n}, & & E_{h^{\prime}}=E \cap \mathbb{R}_{h^{\prime}}^{1+n}, \\
\partial_{0} E_{h^{\prime}} & =\partial_{0} E \cap \mathbb{R}_{h^{\prime}}^{1+n}, & & \Omega_{h^{\prime}}=\Omega \cap \mathbb{R}_{h^{\prime}}^{1+n} .
\end{aligned}
$$

For $z: \Omega_{h^{\prime}} \rightarrow \mathbb{R}, u: \Omega_{h^{\prime}} \rightarrow \mathbb{R}^{n}$ we write $z^{(r, m)}=z\left(t^{(r)}, x^{(m)}\right), u^{(r, m)}=$ $u\left(t^{(r)}, x^{(m)}\right)$ and

$$
\begin{aligned}
& \|z\|_{h^{\prime} . r}=\max \left\{\left|z^{(i, m)}\right|:\left(t^{(i)}, x^{(m)}\right) \in \Omega_{h^{\prime}}, i \leq r\right\}, \\
& \|u\|_{h^{\prime} . r}=\max \left\{\left\|u^{(i, m)}\right\|:\left(t^{(i)}, x^{(m)}\right) \in \Omega_{h^{\prime}}, i \leq r\right\} .
\end{aligned}
$$

Suppose that Assumption $\mathrm{H}[f, \varphi]$ is satisfied with

$$
\sigma(t, p)=\widetilde{L} p, \quad(t, p) \in[0, a] \times \mathbb{R}_{+},
$$

for some $\widetilde{L} \in \mathbb{R}_{+}$. Then the functions $\partial_{x} f, \partial_{w} f, \partial_{q} f$ satisfy the Lipschitz condition with respect to $(w, q)$ and the right hand sides of system (13)-(14) satisfy the Lipschitz condition with respect to the unknown functions with a constant $L(h)$ and

$$
\lim _{h \rightarrow 0} L(h)=\infty .
$$

Suppose that we apply the Euler difference method to solve problem (13)(15) numerically. Then we get a new difference method for problem (1), (2). It follows from (35) that we need additional assumptions on $h_{0}$ and $h$ to get a convergent difference scheme for (1), (2).

It is not our purpose to study all difference methods which can be obtained by a discretization in time of the generalized method of lines. We restrict our attention to the Euler method for (13)-(15).

No attempt has been made here to develop difference schemes generated by the Runge-Kutta methods for (13)-(15). We give numerical examples only.

Suppose that $\left(\delta_{1}, \ldots, \delta_{n}\right)=\delta$ are difference operators defined by (9), (10). Write

$$
\delta z^{(r, m)}=\left(\delta_{1} z^{(r, m)}, \ldots, \delta_{n} z^{(r, m)}\right)=\left(\delta_{1} z^{(m)}\left(t^{(r)}\right), \ldots, \delta_{n} z^{(m)}\left(t^{(r)}\right)\right)
$$

and

$$
\delta_{0} z^{(r, m)}=\frac{1}{h_{0}}\left(z^{(r+1, m)}-z^{(r, m)}\right), \quad \delta_{0} u=\left(\delta_{0} u_{1}, \ldots, \delta_{0} u_{n}\right) .
$$

Let us now define the interpolating operator $T_{h^{\prime}}: \mathbb{F}\left(\Omega_{h^{\prime}}, \mathbb{R}\right) \rightarrow C(\Omega, \mathbb{R})$. Suppose that $z \in \mathbb{F}\left(\Omega_{h^{\prime}}, \mathbb{R}\right)$. Two cases will be distinguished. 
I. Suppose that $(t, x) \in \Omega$ and there is $(r, m) \in \mathbb{Z}^{1+n}$ such that $\left(t^{(r)}, x^{(m)}\right)$, $\left(t^{(r+1)}, x^{(m+1)}\right) \in \Omega_{h^{\prime}}$ and $t^{(r)} \leq t \leq t^{(r+1)}, x^{(m)} \leq x \leq x^{(m+1)}$. We define

$$
\begin{aligned}
T_{h^{\prime}}[z](t, x)= & \left(\frac{t-t^{(i)}}{h_{0}}\right) \sum_{s \in S} z^{(r+1, m+s)}\left(\frac{x-x^{(m)}}{h^{\prime}}\right)^{s}\left(1-\frac{x-x^{(m)}}{h^{\prime}}\right)^{1-s} \\
& +\left(1-\frac{t-t^{(i)}}{h_{0}}\right) \sum_{s \in S} z^{(r, m+s)}\left(\frac{x-x^{(m)}}{h^{\prime}}\right)^{s}\left(1-\frac{x-x^{(m)}}{h^{\prime}}\right)^{1-s} .
\end{aligned}
$$

II. Suppose that $(t, x) \in \Omega$ and $t^{\left(K_{0}\right)}<t \leq a$. There is $m \in \mathbb{Z}^{1+n}$ such that $x^{(m)}, x^{(m+1)} \in[-c, c]$ and $x^{(m)} \leq x \leq x^{(m+1)}$. Write

$$
T_{h^{\prime}}[z](t, x)=T_{h^{\prime}}[z]\left(t^{\left(K_{0}\right)}, x\right) .
$$

Thus we have defined $T_{h^{\prime}}: \Omega \rightarrow \mathbb{R}$. If $u \in \mathbb{F}\left(\Omega_{h^{\prime}}, \mathbb{R}^{n}\right)$ and $u=\left(u_{1}, \ldots, u_{n}\right)$ then we put $T_{h^{\prime}}[u]=\left(T_{h^{\prime}}\left[u_{1}\right], \ldots, T_{h^{\prime}}\left[u_{n}\right]\right)$.

For functions $z \in C(\Omega, \mathbb{R})$ and $u \in C\left(\Omega, \mathbb{R}^{n}\right)$ we put

$$
\begin{aligned}
& \|z\|_{t}=\max \{|z(s, y)|:(s, y) \in \Omega, s \leq t\}, \\
& \|u\|_{t}=\max \{\|u(s, y)\|:(s, y) \in \Omega, s \leq t\} .
\end{aligned}
$$

Lemma 5.1. Suppose that

1) $\widetilde{v}: \Omega \rightarrow \mathbb{R}$ is of class $C^{1}$ and $\widetilde{v}_{h^{\prime}}$ is the restriction of $v$ to $\Omega_{h^{\prime}}$,

2) $h^{\prime} \in H^{\prime}$ and $\widetilde{c} \in \mathbb{R}_{+}$is a constant such that

$$
\left|\partial_{t} \widetilde{v}(t, x)\right| \leq \widetilde{c}, \quad \| \partial_{x_{i}} \widetilde{v}(t, x) \mid \leq \widetilde{c}, 1 \leq i \leq n,(t, x) \in \Omega .
$$

Then

$$
\left\|T_{h^{\prime}}\left[\widetilde{v}_{h^{\prime}}\right]-\widetilde{v}\right\|_{t} \leq \widetilde{c}\left\|h^{\prime}\right\|, \quad t \in\left[-\tau_{0}, K_{0} h_{0}\right] .
$$

The proof of the above lemma is similar to the proof of Theorem 5.27 in [10]. We omit the details.

Set

$$
Q^{(r, m)}[z, u]=\left(t^{(r)}, x^{(m)},\left(T_{h^{\prime}}[z]\right)_{\alpha\left(t^{(r)}, x^{(m)}\right)}, u^{(r, m)}\right) .
$$

We denote by $F_{h^{\prime}}, G_{h^{\prime}}$ the operators defined on $\mathbb{F}\left(\Omega_{h^{\prime}}, \mathbb{R}^{1+n}\right)$ in the following way: if $(z, u) \in \mathbb{F}\left(\Omega_{h^{\prime}}, \mathbb{R}^{1+n}\right)$, then

$$
F_{h^{\prime}}[z, u]^{(r, m)}=f\left(Q^{(r, m)}[z, u]\right)+\partial_{q} f\left(Q^{(r, m)}[z, u]\right) \circ\left(\delta z^{(r, m)}-u^{(r, m)}\right)
$$

and

$$
\begin{aligned}
& G_{h^{\prime}}[z, u]^{(r, m)} \\
& \quad=\partial_{x} f\left(Q^{(r, m)}[z, u]\right)+\partial_{w} f\left(Q^{(r, m)}[z, u]\right)\left(T_{h^{\prime}}[u]\right)_{\alpha^{(r, m)}} \star \partial_{x} \alpha^{\prime(r, m)} \\
& \quad+\partial_{q} f\left(Q^{(r, m)}[z, u]\right) \star\left[\delta u^{(r, m)}\right]^{T}
\end{aligned}
$$


where

$$
\begin{aligned}
& \partial_{w} f\left(Q^{(r, m)}[z, u]\right)\left(T_{h^{\prime}}[u]\right)_{\alpha^{(r, m)}} \\
& \quad=\left(\partial_{w} f\left(Q^{(r, m)}[z, u]\right)\left(T_{h^{\prime}}\left[u_{1}\right]\right)_{\alpha^{(r, m)}}, \ldots, \partial_{w} f\left(Q^{(r, m)}[z, u]\right)\left(T_{h^{\prime}}\left[u_{n}\right]\right)_{\alpha^{(r, m)}}\right) .
\end{aligned}
$$

An application of the Euler difference method to (13)-(15) leads to the difference equations

$$
\begin{aligned}
& \delta_{0} z^{(r, m)}=F_{h^{\prime}}[z, u]^{(r, m)}, \\
& \delta_{0} u^{(r, m)}=G_{h^{\prime}}[z, u]^{(r, m)},
\end{aligned}
$$

with initial boundary conditions

$$
z^{(r, m)}=\varphi_{h^{\prime}}^{(r, m)}, \quad u^{(r, m)}=\psi_{h^{\prime}}^{(r, m)} \quad \text { on } E_{0 . h^{\prime}} \cup \partial_{0} E_{h^{\prime}}
$$

where $\varphi_{h^{\prime}}: E_{0 . h^{\prime}} \cup \partial_{0} E_{h^{\prime}} \rightarrow \mathbb{R}$ and $\psi_{h^{\prime}}: E_{0 . h^{\prime}} \cup \partial_{0} E_{h^{\prime}} \rightarrow \mathbb{R}^{n}$ are given functions. Put

$$
\widetilde{E}_{h^{\prime} . r}=\left\{\left(t^{(r)}, x^{(m)}\right) \in E_{h^{\prime} . r}: r \leq K_{0}-1\right\} .
$$

Now we state a theorem on the convergence of the generalized Euler method.

TheOREM 5.2. Suppose that Assumptions $\mathrm{H}[\alpha], \mathrm{H}[f, \varphi]$ are satisfied and

1) $h^{\prime}=\left(h_{0}, h\right) \in H^{\prime}$ and

$$
1-h_{0} \sum_{i=1}^{n} \frac{1}{h_{i}}\left|\partial_{q_{i}} f(P)\right| \geq 0
$$

for all $P=(t, x, w, q) \in \Sigma$,

2) the functions $\left(z_{h^{\prime}}, u_{h^{\prime}}\right)$, where $z_{h^{\prime}}: \Omega_{h} \rightarrow \mathbb{R}, u_{h^{\prime}}: \Omega_{h} \rightarrow \mathbb{R}^{n}$, satisfy (38)-(40), and there is $\beta_{0}\left(h^{\prime}\right): H^{\prime} \rightarrow \mathbb{R}_{+}$such that

$$
\left|\varphi^{(r, m)}-\varphi_{h^{\prime}}^{(r, m)}\right| \leq \beta_{0}\left(h^{\prime}\right), \quad\left\|\psi^{(r, m)}-\psi_{h^{\prime}}^{(r, m)}\right\| \leq \beta_{0}\left(h^{\prime}\right) \quad \text { on } E_{0 . h^{\prime}} \cup \partial_{0} E_{h^{\prime}},
$$

3) $v: \Omega \rightarrow \mathbb{R}$ is a solution of (1), (2) and $v$ is of class $C^{2}$ on $\Omega$.

Then there are $\varepsilon_{0}>0$ and $\beta: H^{\prime} \rightarrow \mathbb{R}_{+}$such that for $\left\|h^{\prime}\right\|<\varepsilon_{0}$ we have

$$
\max \left\{\left\|v_{h^{\prime}}-z_{h^{\prime}}\right\|_{h^{\prime} . r},\left\|\partial_{x} v_{h^{\prime}}-u_{h^{\prime}}\right\|_{h^{\prime} . r}\right\} \leq \beta\left(h^{\prime}\right), \quad 0 \leq r \leq K_{0},
$$

where $v_{h^{\prime}}$ and $\partial_{x} v_{h^{\prime}}$ are the restrictions of $v$ and $\partial_{x} v$ to $\Omega_{h^{\prime}}$.

Proof. We have divided the proof into a sequence of steps.

I. Suppose that $\Phi: \Omega_{h^{\prime}} \rightarrow \mathbb{R}$ satisfies the difference equation

$$
\delta_{0} \Phi^{(r, m)}=\partial_{q} f\left(Q^{(r, m)}\left[z_{h^{\prime}}, u_{h^{\prime}}\right]\right) \circ \delta \Phi^{(r, m)}+\Xi^{(r, m)}
$$

on $\widetilde{E}_{h^{\prime} . r}$ where $\Xi: \widetilde{E}_{h^{\prime} . r} \rightarrow \mathbb{R}$ is a given function. We prove that

$$
\left|\Phi^{(r+1, m)}\right| \leq\|\Phi\|_{h^{\prime} . r}+h_{0}\left|\Xi^{(r, m)}\right| .
$$


Put

$$
\begin{aligned}
& J^{+}[r, m]=\left\{j \in\{1, \ldots, n\}: \partial_{q_{j}} f\left(Q^{(r, m)}\left[z_{h^{\prime}}, u_{h^{\prime}}\right]\right) \geq 0\right\} \\
& J^{-}[r, m]=\{1, \ldots, n\} \backslash J^{+}[r, m] .
\end{aligned}
$$

Consider the operator $W_{h^{\prime}}: \mathbb{F}\left(\Omega_{h^{\prime}}, \mathbb{R}\right) \rightarrow \mathbb{F}\left(\widetilde{E}_{h^{\prime} . r}, \mathbb{R}\right)$ defined by

$$
\begin{aligned}
W_{h^{\prime}}[\theta]^{(r, m)}= & \theta^{(r, m)}\left[1-h_{0} \sum_{j=1}^{n} \frac{1}{h_{j}}\left|\partial_{q_{j}} f\left(Q^{(r, m)}\left[z_{h^{\prime}}, u_{h^{\prime}}\right]\right)\right|\right] \\
& +h_{0} \sum_{j \in J^{+}[r, m]} \frac{1}{h_{j}} \partial_{q_{j}} f\left(Q^{(r, m)}\left[z_{h^{\prime}}, u_{h^{\prime}}\right]\right) \theta^{\left(r, m+e_{j}\right)} \\
& -h_{0} \sum_{j \in J^{-}[r, m]} \frac{1}{h_{j}} \partial_{q_{j}} f\left(Q^{(r, m)}\left[z_{h^{\prime}}, u_{h^{\prime}}\right]\right) \theta^{\left(r, m-e_{j}\right)}
\end{aligned}
$$

for all $\theta \in \mathbb{F}\left(\Omega_{h^{\prime}}, \mathbb{R}\right)$ and $\left(t^{(r)}, x^{(m)}\right) \in \widetilde{E}_{h^{\prime} . r}$. It follows from (9), (10) and condition 2) of Assumption $\mathrm{H}_{0}[f, \varphi]$ that relation (43) is equivalent to

$$
\Phi^{(r+1, m)}=W_{h^{\prime}}[\Phi]^{(r, m)}+h_{0} \Xi^{(r, m)} .
$$

According to assumption (41), we have

$$
\left|W_{h^{\prime}}[\Phi]^{(r, m)}\right| \leq\|\Phi\|_{h^{\prime} . r} \quad \text { for }\left(t^{(r)}, x^{(m)}\right) \in \widetilde{E}_{h^{\prime} . r}
$$

and (44) is proved.

II. Define $w: \Omega \rightarrow \mathbb{R}^{n}$ by $w=\partial_{x} v$. Then $(v, w)$ satisfies the quasilinear system (16), (17) and the initial condition (18). Set

$$
P^{(r, m)}[v, w]=U\left[v, w ; t^{(r)}, x^{(m)}\right], \quad \Phi^{(r, m)}=\left(v_{h^{\prime}}-z_{h^{\prime}}\right)^{(r, m)} .
$$

It follows from (16) and from (38) that the function $\Phi$ satisfies (43) with

$$
\begin{aligned}
& \Xi^{(r, m)}=\delta_{0} v_{h^{\prime}}^{(r, m)}-\partial_{t} v^{(r, m)} \\
& +f\left(P^{(r, m)}[v, w]\right)-f\left(Q^{(r, m)}\left[z_{h^{\prime}}, u_{h^{\prime}}\right]\right)+\partial_{q} f\left(Q^{(r, m)}\left[z_{h^{\prime}}, u_{h^{\prime}}\right]\right) \circ\left[u_{h^{\prime}}^{(r, m)}-\delta v_{h^{\prime}}^{(r, m)}\right] .
\end{aligned}
$$

It follows from (28), Lemma 5.1 and Assumption $\mathrm{H}[f, \varphi]$ that there is $\gamma_{0}$ : $H^{\prime} \rightarrow \mathbb{R}_{+}$such that

$$
\left|\Xi^{(r, m)}\right| \leq B\left(\left\|v_{h^{\prime}}-z_{h^{\prime}}\right\|_{h^{\prime} . r}+2 n\left\|\left(w_{h^{\prime}}-u_{h^{\prime}}\right)^{(r, m)}\right\|\right)+\gamma_{0}\left(h^{\prime}\right),
$$

and $\lim _{h^{\prime} \rightarrow 0} \gamma_{0}\left(h^{\prime}\right)=0$. Thus

$$
\begin{aligned}
& \left|\left(v_{h^{\prime}}-z_{h^{\prime}}\right)^{(r+1, m)}\right| \\
& \quad \leq\left(1+h_{0} B\right)\left\|v_{h^{\prime}}-z_{h^{\prime}}\right\|_{h^{\prime} . r}+2 n B h_{0}\left\|\left(w_{h^{\prime}}-u_{h^{\prime}}\right)^{(r, m)}\right\|+h_{0} \gamma_{0}\left(h^{\prime}\right) .
\end{aligned}
$$

III. Now we define

$$
\Phi^{(r, m)}=\left(w_{h^{\prime} . i}-u_{h^{\prime} . i}\right)^{(r, m)}
$$


where $i, 1 \leq i \leq n$, is fixed. We see from (17), (39) that the function $\Phi$ so defined satisfies (43) with

$$
\begin{aligned}
\Xi^{(r, m)}= & \delta_{0} w_{h^{\prime} . i}^{(r, m)}-\partial_{t} w_{i}^{(r, m)}+\partial_{q} f\left(P^{(r, m)}[v, w]\right) \circ\left(\partial_{x} w_{i}-\delta w_{h^{\prime} . i}\right)^{(r, m)} \\
& +\partial_{x_{i}} f\left(P^{(r, m)}[v, w]\right)-\partial_{x_{i}} f\left(Q^{(r, m)}\left[z_{h^{\prime}}, u_{h^{\prime}}\right]\right) \\
& +\partial_{w} f\left(P^{(r, m)}[v, w]\right) w_{\alpha^{(r, m)}} \circ \partial_{x_{i}} \alpha^{(r, m)} \\
& -\partial_{w} f\left(Q^{(r, m)}\left[z_{h^{\prime}}, u_{h^{\prime}}\right]\right)\left(T_{h^{\prime}}\left[u_{h^{\prime}}\right]\right)_{\alpha^{(r, m)}} \circ \partial_{x_{i}} \alpha^{\prime(r, m)} \\
& +\left(\partial_{q} f\left(P^{(r, m)}[v, w]\right)-\partial_{q} f\left(Q^{(r, m)}\left[z_{h^{\prime}}, u_{h^{\prime}}\right]\right)\right) \delta w_{h^{\prime} . i}^{(r, m)} .
\end{aligned}
$$

It follows from (28), (29), Lemma 5.1 and Assumption $\mathrm{H}[f, \varphi]$ that there is $\gamma: H^{\prime} \rightarrow \mathbb{R}_{+}$such that

$$
\begin{aligned}
\left|\Xi^{(r, m)}\right| \leq & B \widetilde{s}\left\|w_{h^{\prime}}-u_{h^{\prime}}\right\|_{h^{\prime} . r} \\
& +(1+n \widetilde{c}+\widetilde{s}) \sigma\left(t^{(r)}, \max \left\{\left\|v_{h^{\prime}}-z_{h^{\prime}}\right\|_{h^{\prime} . r},\left\|\left(w_{h^{\prime}}-u_{h^{\prime}}\right)^{(r, m)}\right\|\right\}\right)+\gamma\left(h^{\prime}\right),
\end{aligned}
$$

and $\lim _{h^{\prime} \rightarrow 0} \gamma\left(h^{\prime}\right)=0$. Thus

$$
\begin{aligned}
& \text { (48) }\left\|\left(w_{h^{\prime}}-u_{h^{\prime}}\right)^{(r+1, m)}\right\| \leq\left(1+h_{0} B \widetilde{s}\right)\left\|w_{h^{\prime}}-u_{h^{\prime}}\right\|_{h^{\prime} . r} \\
& +h_{0}(1+n \widetilde{c}+\widetilde{s c}) \sigma\left(t^{(r)}, \max \left\{\left\|v_{h^{\prime}}-z_{h^{\prime}}\right\|_{h^{\prime} . r},\left\|\left(w_{h^{\prime}}-u_{h^{\prime}}\right)^{(r, m)}\right\|\right\}\right)+h_{0} \gamma\left(h^{\prime}\right) .
\end{aligned}
$$

IV. Write

$$
\xi_{h^{\prime}}^{(r)}=\max \left\{\left\|v_{h^{\prime}}-z_{h^{\prime}}\right\|_{h^{\prime} . r},\left\|w_{h^{\prime}}-u_{h^{\prime}}\right\|_{h^{\prime} . r}\right\}, \quad 0 \leq r \leq K_{0} .
$$

According to the estimates (44)-(48) we have

$$
\xi_{h^{\prime}}^{(r+1)} \leq \xi_{h^{\prime}}^{(r)}+h_{0}\left(\widetilde{a} \xi_{h^{\prime}}^{(r)}+\bar{p} \sigma\left(t^{(r)}, \xi_{h^{\prime}}^{(r)}\right)+\bar{\gamma}\left(h^{\prime}\right)\right), \quad 0 \leq r \leq K_{0}-1,
$$

where

$$
\widetilde{a}=B \max \{2 n+1, \widetilde{s}\}, \quad \bar{p}=1+n \widetilde{c}+\widetilde{s c}, \quad \bar{\gamma}\left(h^{\prime}\right)=\gamma_{0}\left(h^{\prime}\right)+\gamma\left(h^{\prime}\right) .
$$

Consider the Cauchy problem

$$
\omega^{\prime}(t)=\widetilde{a} \omega(t)+\bar{p} \sigma(t, \omega(t))+\bar{\gamma}\left(h^{\prime}\right), \quad \omega(0)=\beta_{0}\left(h^{\prime}\right) .
$$

It follows from Assumption $\mathrm{H}[\sigma]$ that there is $\varepsilon_{0}>0$ such that for $\left\|h^{\prime}\right\| \leq \varepsilon_{0}$ there exists the maximum solution $\omega_{h^{\prime}}$ of $(51)$ and $\omega_{h^{\prime}}$ is defined on $[0, a]$. Moreover, we have

$$
\lim _{h^{\prime} \rightarrow 0} \omega_{h^{\prime}}(t)=0 \quad \text { uniformly on }[0, a] .
$$

The function $\omega_{h^{\prime}}$ satisfies the difference inequality

$$
\omega_{h^{\prime}}^{(r+1)} \geq \omega_{h^{\prime}}^{(r)}+h_{0}\left(\widetilde{a} \omega_{h^{\prime}}^{(r)}+\bar{p} \sigma\left(t^{(r)}, \omega_{h^{\prime}}^{(r)}\right)+\bar{\gamma}\left(h^{\prime}\right)\right), \quad 0 \leq r \leq K_{0}-1 .
$$

By the above inequality and (49) we have

$$
\xi_{h^{\prime}}^{(r)} \leq \omega_{h^{\prime}}^{(r)} \quad \text { for } 0 \leq r \leq K_{0} .
$$

Thus we get (42) for $\beta\left(h^{\prime}\right)=\omega_{h^{\prime}}(a)$. This completes the proof. 
REMARK 5.3. Suppose that all the assumptions of Theorem 5.2 are satisfied with $\sigma(t, \xi)=L \xi$ on $[0, a] \times \mathbb{R}_{+}$for some $L \in \mathbb{R}_{+}$. Then (42) holds true with

$$
\beta\left(h^{\prime}\right)= \begin{cases}\beta_{0}\left(h^{\prime}\right) e^{\bar{L} a}+\bar{\gamma}\left(h^{\prime}\right) \frac{e^{\bar{L} a}-1}{\bar{L}} & \text { if } \bar{L}>0, \\ \beta_{0}\left(h^{\prime}\right)+a \bar{\gamma}\left(h^{\prime}\right) & \text { if } \bar{L}=0,\end{cases}
$$

where $\bar{L}=\widetilde{a}+L \bar{p}$ and $\widetilde{a}, \bar{p}, \bar{\gamma}$ are given by (50).

\section{Numerical experiments}

EXAMPLE 6.1. Let $n=2$ and

$$
\begin{gathered}
E=[0,1 / 2] \times(-\pi / 2, \pi / 2) \times(-\pi / 2, \pi / 2), \\
E_{0}=\{0\} \times[-\pi / 2, \pi / 2], \\
\partial_{0} E=(0,1 / 2] \times([-\pi / 2, \pi / 2] \times[-\pi / 2, \pi / 2] \backslash(-\pi / 2, \pi / 2) \times(-\pi / 2, \pi / 2)) .
\end{gathered}
$$

Consider the mixed problem

$$
\begin{gathered}
\text { (53) } \partial_{t} z(t, x, y)=x\left[\partial_{x} z(t, x, y)+\sin \left(\partial_{x} z(t, x, y)+\frac{1}{2} \int_{-x}^{x} z(t, s, y) d s\right)\right] \\
+y\left[\partial_{y} z(t, x, y)-\cos \left(\partial_{y} z(t, x, y)+\frac{1}{2} \int_{-y}^{y} z(t, x, s) d s\right)\right]+z(t, x, y)+f(t, x, y), \\
(54) \quad z(t, x, y)=0 \quad \text { for }(t, x, y) \in E_{0} \cup \partial_{0} E
\end{gathered}
$$

where

$$
f(t, x)=e^{t}(\cos x \cos y+x t \sin x \cos y+y t \cos x \sin y)+y .
$$

The solution of the problem is given by $v(t, x)=t e^{t} \cos x \cos y$. The Lax scheme is a classical difference method for (53), (54) and it has the form

$$
\begin{aligned}
& \delta_{0} z^{(r, m)}=x^{\left(m_{1}\right)}\left(\delta_{1} z^{(r, m)}+\sin \left(\delta_{1} z^{(r, m)}+\frac{1}{2} I_{1} z^{(r, m)}\right)\right) \\
& +y^{\left(m_{2}\right)}\left(\delta_{2} z^{(r, m)}-\cos \left(\delta_{2} z^{(r, m)}+\frac{1}{2} I_{2} z^{(r, m)}\right)\right)+z^{(r, m)}+f^{(r, m)}, \\
& z^{\left(0, m_{1}, m_{2}\right)}=z^{\left(r, N_{1}, m_{2}\right)}=z^{\left(r,-N_{1}, m_{2}\right)}=z^{\left(r, m_{1}, N_{2}\right)}=z^{\left(r, m_{1},-N_{2}\right)}=0
\end{aligned}
$$

for $t^{(r)} \in[0, a], x^{\left(m_{1}\right)} \in[-\pi / 2, \pi / 2], y^{\left(m_{2}\right)} \in[-\pi / 2, \pi / 2]$, where $m=$ $\left(m_{1}, m_{2}\right)$ and

$$
\begin{gathered}
\delta_{0} z^{(r, m)}=\frac{1}{h_{0}}\left[z^{(r+1, m)}-\Delta z^{(r, m)}\right], \\
\Delta z^{(r, m)}=\frac{1}{4}\left(z^{\left(r, m_{1}-1, m_{2}\right)}+z^{\left(r, m_{1}+1, m_{2}\right)}+z^{\left(r, m_{1}, m_{2}-1\right)}+z^{\left(r, m_{1}, m_{2}+1\right)}\right)
\end{gathered}
$$


and

$$
\begin{aligned}
& \delta_{1} z^{(r, m)}=\frac{1}{2 h_{1}}\left[z^{\left(r, m_{1}+1, m_{2}\right)}-z^{\left(r, m_{1}-1, m_{2}\right)}\right] \\
& \delta_{2} z^{(r, m)}=\frac{1}{2 h_{2}} g\left[z^{\left(r, m_{1}, m_{2}+1\right)}-z^{\left(r, m_{1}, m_{2}-1\right)}\right], \\
& I_{1} z^{(r, m)}=\operatorname{sign}\left(m_{1}\right) \frac{1}{2 h_{1}} \sum_{k=-m_{1}}^{m_{1}-1}\left(z^{\left(r, k, m_{2}\right)}+z^{\left(r, k+1, m_{2}\right)}\right), \\
& I_{2} z^{(r, m)}=\operatorname{sign}\left(m_{2}\right) \frac{1}{2 h_{2}} \sum_{k=-m_{2}}^{m_{2}-1}\left(z^{\left(r, m_{1}, k\right)}+z^{\left(r, m_{2}, k+1\right)}\right) .
\end{aligned}
$$

The convergence of the method (55), (56) follows from [9]. Denote by $z_{h}: E_{h} \rightarrow \mathbb{R}$ the solution of problem (55), (56) and by $\bar{z}_{h}: E_{h} \rightarrow \mathbb{R}$, $\bar{u}_{h}: E_{h} \rightarrow \mathbb{R}^{2}$ the solution given by the generalized Euler method for problem (53), (54).

Suppose that $t^{(r)}$ is fixed for some $0 \leq r \leq K$. Then we put

$$
\begin{aligned}
& \varepsilon_{h}^{(r)}=\max \left\{\left|z_{h}^{(r, m)}-v^{(r, m)}\right|:-N \leq m \leq N\right\} \\
& \nu_{h}^{(r)}=\frac{1}{\left(2 N_{1}-1\right)\left(2 N_{2}-1\right)} \sum_{-N \leq m \leq N}\left|z_{h}^{(r, m)}-v^{(r, m)}\right|,
\end{aligned}
$$

where $N=\left(N_{1}, N_{2}\right)$. The numbers $\varepsilon_{h}^{(r)}$ and $\nu_{h}^{(r)}$ can be called the maximal and average errors of the classical method for fixed $t^{(r)}$. In a similar way we define the maximal and average errors $\bar{\varepsilon}_{h}^{(r)}, \bar{\nu}_{h}^{(r)}$ for the generalized Euler method.

We put $h_{0}=0.001, h_{1}=h_{2}=0.01$ and we have the following experimental values for the above defined errors.

Table 1. Maximal errors $\varepsilon_{h}, \bar{\varepsilon}_{h}$ and average errors $\nu_{h}, \bar{\nu}_{h}$

\begin{tabular}{lllll}
\hline$t^{(r)}$ & $\varepsilon_{h}^{(r)}$ & $\bar{\varepsilon}_{h}^{(r)}$ & $\nu_{h}^{(r)}$ & $\bar{\nu}_{h}^{(r)}$ \\
0.1 & $1.40 \cdot 10^{-3}$ & $9.61 \cdot 10^{-5}$ & $5.39 \cdot 10^{-4}$ & $4.91 \cdot 10^{-5}$ \\
0.2 & $6.03 \cdot 10^{-3}$ & $3.36 \cdot 10^{-4}$ & $2.19 \cdot 10^{-3}$ & $1.58 \cdot 10^{-4}$ \\
0.3 & $1.47 \cdot 10^{-2}$ & $7.64 \cdot 10^{-4}$ & $5.09 \cdot 10^{-3}$ & $3.25 \cdot 10^{-4}$ \\
0.4 & $2.85 \cdot 10^{-2}$ & $1.43 \cdot 10^{-3}$ & $9.39 \cdot 10^{-3}$ & $5.82 \cdot 10^{-4}$ \\
0.5 & $4.85 \cdot 10^{-2}$ & $4.86 \cdot 10^{-3}$ & $1.53 \cdot 10^{-2}$ & $1.05 \cdot 10^{-3}$ \\
\hline
\end{tabular}

Note that $\bar{\varepsilon}_{h}^{(r)}<\varepsilon_{h}^{(r)}$ and $\bar{\nu}_{h}^{(r)}<\nu_{h}^{(r)}$ for all values of $t^{(r)}$.

EXAMPle 6.2. Let $n=1$. Consider the mixed problem

$$
\begin{aligned}
\partial_{t} z(t, x)= & x\left(\partial_{x} z(t, x)-\cos \left(\partial_{x} z(t, x)-t x z(t, x)\right)\right) \\
& +f(t, x)\left[z\left(t, \frac{x+1}{2}\right)+z\left(t, \frac{x-1}{2}\right)\right],
\end{aligned}
$$




$$
z(0, x)=1 \quad \text { for } x \in[-1,1], \quad z(t,-1)=z(t, 1)=1 \quad \text { for } t \in[0, a],
$$

where

$$
f(t, x)=\left[x+\frac{1}{2}\left((1-2 t) x^{2}-1\right) e^{\frac{1}{2} t\left(x^{2}-1\right)}\right]\left[e^{\frac{1}{8} t(x-1)(x+3)}+e^{\frac{1}{8} t(x+1)(x-3)}\right]^{-1} .
$$

The solution of this problem is given by $v(t, x)=e^{\frac{1}{2} t\left(x^{2}-1\right)}$.

Let $z_{h}, h=\left(h_{0}, h_{1}\right)$, denote the numerical solution of $(57),(58)$ which is obtained by the classical Lax scheme (see [10]).

The quasilinear differential functional system corresponding to (57) has the form

$$
\begin{aligned}
\partial_{t} z(t, x)= & x(u(t, x)-\cos (u(t, x)-t x z(t, x))) \\
& +f(t, x)\left[z\left(t, \frac{x+1}{2}\right)+z\left(t, \frac{x-1}{2}\right)\right] \\
& +x(1+\sin (u(t, x)-t x z(t, x)))\left(\partial_{x} z(t, x)-u(t, x)\right), \\
\partial_{t} u(t, x)= & u(t, x)-\cos (u(t, x)-t x z(t, x)) \\
& -t x(x+z(t, x)) \sin (u(t, x)-t x z(t, x)) \\
& +\partial_{x} f(t, x)\left[z\left(t, \frac{x+1}{2}\right)+z\left(t, \frac{x-1}{2}\right)\right] \\
& +\frac{1}{2} f(t, x)\left[u\left(t, \frac{x+1}{2}\right)+u\left(t, \frac{x-1}{2}\right)\right] \\
& +x(1+\sin (u(t, x)-t x z(t, x))) \partial_{x} u(t, x) .
\end{aligned}
$$

We apply the numerical method of lines for the above system and then the Runge-Kutta method of the 4-th order for the system of ordinary differential equations.

Denote by $\bar{z}_{h}: E_{h} \rightarrow \mathbb{R}, \bar{u}_{h}: E_{h} \rightarrow \mathbb{R}^{2}$ the solution given by the generalized method of lines and Runge-Kutta method for problem (57), (58). Suppose that $t^{(r)} \in[0, a]$ is fixed. Then we put

$$
\begin{aligned}
\varepsilon_{h}^{(r)} & =\max \left\{\left|z_{h}^{(r, m)}-v^{(r, m)}\right|:-N \leq m \leq N\right\}, \\
\nu_{h}^{(r)} & =\frac{1}{2 N-1} \sum_{-N \leq m \leq N}\left|z_{h}^{(r, m)}-v^{(r, m)}\right| .
\end{aligned}
$$

The numbers $\varepsilon_{h}^{(r)}$ and $\nu_{h}^{(r)}$ can be called the maximal and average errors of the classical method for fixed $t^{(r)}$. In a similar way we define the maximal and average errors $\bar{\varepsilon}_{h}^{(r)}, \bar{\nu}_{h}^{(r)}$ for the generalized method of lines and Runge-Kutta method.

We put $a=1, b=1, h_{0}=0.0001, h_{1}=0.01$ and we have the following experimental values for the above defined errors. 
Table 2. Maximal errors $\varepsilon_{h}, \bar{\varepsilon}_{h}$ and average errors $\nu_{h}, \bar{\nu}_{h}$

\begin{tabular}{lllll}
\hline$t^{(r)}$ & $\varepsilon_{h}^{(r)}$ & $\bar{\varepsilon}_{h}^{(r)}$ & $\nu_{h}^{(r)}$ & $\bar{\nu}_{h}^{(r)}$ \\
0.2 & $9.17 \cdot 10^{-3}$ & $9.78 \cdot 10^{-5}$ & $7.82 \cdot 10^{-3}$ & $4.94 \cdot 10^{-5}$ \\
0.4 & $3.31 \cdot 10^{-2}$ & $3.62 \cdot 10^{-4}$ & $2.70 \cdot 10^{-2}$ & $1.81 \cdot 10^{-4}$ \\
0.6 & $6.68 \cdot 10^{-2}$ & $7.54 \cdot 10^{-4}$ & $5.33 \cdot 10^{-2}$ & $3.81 \cdot 10^{-4}$ \\
0.8 & $1.06 \cdot 10^{-1}$ & $1.24 \cdot 10^{-3}$ & $8.39 \cdot 10^{-2}$ & $6.38 \cdot 10^{-4}$ \\
1.0 & $1.48 \cdot 10^{-1}$ & $1.81 \cdot 10^{-3}$ & $1.17 \cdot 10^{-1}$ & $9.44 \cdot 10^{-4}$ \\
\hline
\end{tabular}

Note that $\bar{\varepsilon}_{h}^{(r)}<\varepsilon_{h}^{(r)}$ and $\bar{\nu}_{h}^{(r)}<\nu_{h}^{(r)}$ for all values of $t^{(r)}$.

Thus we see that the errors of the classical method are larger than the errors of the generalized method of lines. This is due to the fact that the approximation of the spatial derivative of $z$ in the generalized method of lines is better than the respective approximation in the classical method. The method described in this paper may be applied for numerical solution of first order nonlinear differential equations with deviated variables.

\section{References}

[1] A. Baranowska and Z. Kamont, Numerical method of lines for first order partial differential-functional equations, Z. Anal. Anwend. 21 (2002), 949-962.

[2] S. Cinquini, On hyperbolic systems of (nonlinear) partial differential equations in several independent variables, Ann. Mat. Pura Appl. 120 (1979), 201-214 (in Italian).

[3] M. Cinquini Cibrario, A class of systems of nonlinear partial differential equations in several independent variables, ibid. 140 (1985), 223-253 (in Italian).

[4] M. Dehghan, Numerical procedures for a boundary value problem with a non-linear boundary condition, Appl. Math. Comput. 147 (2004), 291-306.

[5] I. Györi, On the method of lines for the solutions of nonlinear partial differential equations, Akad. Nauk SSSR, Otdel Vychisl. Mat., Moscow, 1987.

[6] —, The method of lines for the solutions of some nonlinear partial differential equations, Comput. Math. Appl. 15 (1998), 635-648.

[7] W. Hundsdorfer and J. Verwer, Numerical Solution of Time-Dependent AdvectionDiffusion-Reaction Equations, Springer Ser. Comput. Math. 33, Springer, Berlin, 2003.

[8] D. Jaruszewska-Walczak and Z. Kamont, Existence of solutions of first order partial differential-functional equations via the method of lines, Serdica 16 (1990), 104-114.

[9] Z. Kamont, Finite difference approximations for first order partial differential functional equations, Ukrain. Math. J. 46 (1994), 985-996.

[10] - , Hyperbolic Fuctional Differential Inequalities and Applications, Kluwer, Dordrecht, 1999.

[11] —, Functional differential and difference inequalities with impulses, Mem. Differential Equations Math. Phys. 24 (2001), 5-82.

[12] Z. Kamont and S. Zacharek, Lines method approximations to the initial boundary value problem of Neumann type for parabolic differential functional equations, Comment. Math. Prace Mat. 30 (1981), 317-330. 
[13] H. Leszczyński, On the method of lines for a non-linear heat equation with functional dependence, Ann. Polon. Math 69 (1998), 61-74.

[14] W. E. Schiesser, The Numerical Method of Lines. Integration of Partial Differential Equations, Acad. Press, San Diego, 1991.

[15] K. Schmitt, R. C. Thompson and W. Walter, Existence of solutions of a nonlinear boundary value problem via the method of lines, Nonliear Anal. 2 (1978), 519-535.

[16] A. Vande Wouwer, Ph. Saucez and W. E. Schiesser, Adaptive Method of Lines, Chapman and Hall/CRC, Boca Raton, 2001.

[17] D. M. Wei, Existence, uniqueness and numerical analysis of solutions of a quasilinear parabolic problem, SIAM J. Numer. Anal. 29 (1992), 484-497.

[18] K. Zlateva, Method of lines for parabolic equations with dynamical boundary conditions, Math. Balkanica (N.S.) 14 (2000), 275-290.

Institute of Mathematics

University of Gdańsk

Wit Stwosz St. 57

80-952 Gdańsk, Poland

E-mail: Wojciech.Czernous@math.univ.gda.pl

Received 20.1.2005

and in final form 17.7.2006 